\title{
The Spectral Correlation Function of Molecular Clouds: A Statistical Test for Theoretical Models
}

\section{Citation}

Padoan, Paolo, Alyssa A. Goodman, and Mika Juvela. 2003. "The Spectral Correlation Function of Molecular Clouds: A Statistical Test for Theoretical Models." The Astrophysical Journal 588 (2): 881-93. https://doi.org/10.1086/374240.

\section{Permanent link}

http://nrs.harvard.edu/urn-3:HUL.InstRepos:41393355

\section{Terms of Use}

This article was downloaded from Harvard University's DASH repository, and is made available under the terms and conditions applicable to Other Posted Material, as set forth at http:// nrs.harvard.edu/urn-3:HUL.InstRepos:dash.current.terms-of-use\#LAA

\section{Share Your Story}

The Harvard community has made this article openly available.

Please share how this access benefits you. Submit a story.

Accessibility 


\title{
THE SPECTRAL CORRELATION FUNCTION OF MOLECULAR CLOUDS: A STATISTICAL TEST FOR THEORETICAL MODELS
}

\author{
Paolo Padoan, ${ }^{1}$ Alyssa A. Goodman, ${ }^{2}$ and Mika Juvela ${ }^{3}$ \\ Received 2002 October 21; accepted 2003 January 16
}

\begin{abstract}
We compute the spectral correlation function (SCF) of ${ }^{13} \mathrm{CO} J=1-0$ maps of molecular cloud complexes. The SCF is a power law over approximately an order of magnitude in spatial separation in every map. The power-law slope of the SCF, $\alpha$, its normalization, $S_{0}(1 \mathrm{pc})$, and the spectral line width averaged over the whole map, $\sigma_{v}$, are computed for all the observational maps. The values of $\alpha, S_{0}(1 \mathrm{pc})$, and $\sigma_{v}$ are combined to obtain empirical correlations to be used as tests for theoretical models of molecular clouds. Synthetic spectral maps are computed from different theoretical models, including solutions of the magnetohydrodynamic (MHD) equations with different values of the rms Mach number of the flow and stochastic models with different power spectra of the velocity field. In order to compute the radiative transfer from the MHD models, it is necessary to assign the models a physical scale and a physical density. When these assignments are made according to Larson-type relations, the best fit to the observational correlations is obtained. Unphysical stochastic models are instead ruled out by the empirical correlations. MHD models with equipartition of magnetic and kinetic energy of turbulence do not reproduce the observational data when their average magnetic field is oriented approximately parallel to the line of sight.
\end{abstract}

Subject headings: ISM: individual (Perseus, Rosette Molecular Complex, Taurus Molecular Cloud) ISM: kinematics and dynamics — radio lines: ISM — turbulence

\section{INTRODUCTION}

Molecular clouds are observed through the emission of a number of molecular transitions, which provide a wealth of information about their chemical composition, gas temperature and density, magnetic field strength, fractional ionization, structure, and kinematics. This information is essential to our understanding of the process of star formation.

The interpretation of molecular emission line maps is not always unique. The main source of uncertainty is the absence of the third spatial dimension (along the line of sight) in the observational data. Statistical properties of the velocity and density distributions along the line of sight are difficult to disentangle. Furthermore, the components of the gas velocity on the plane of the sky are unknown.

Two-dimensional images of molecular clouds are usually converted into three-dimensional " objects" using the radial velocity instead of the third spatial dimension. This method can be useful to separate individual mass condensations from each other, since it is conceivable that their relative velocity is larger than their internal one. However, velocity blending or the lack of well-defined condensations along the line of sight may cause significant uncertainties (Issa, MacLaren, \& Wolfendale 1990; Adler \& Roberts 1992; Ballesteros-Paredes, Vázquez-Semadeni, \& Scalo 1999; Pichardo et al. 2000; Ostriker, Stone, \& Gammie 2001; Lazarian et al. 2001; Ballesteros-Paredes \& Mac Low 2002).

Because of the difficulty of a direct interpretation of the observational data, a "forward approach" that starts from a rather general theoretical model and synthesizes its obser-

\footnotetext{
${ }^{1}$ Jet Propulsion Laboratory, California Institute of Technology, 4800 Oak Grove Drive, MS 169-506, Pasadena, CA 91109-8099; padoan@ jpl.nasa.gov.

${ }_{2}^{2}$ Department of Astronomy, Harvard University, 60 Garden Street, Cambridge, MA 02138.

${ }^{3}$ Helsinki University Observatory, University of Helsinki, Tähtitorninmäki, P.O. Box 14, SF-00014, Finland.
}

vational properties can be more instructive. Different models may sometimes satisfy the same set of observational constraints, but they should also provide guidance for further observational studies that could help select the correct model.

Ideally, numerical models to be compared with observed spectral line data cubes should be based on the numerical solutions of the magnetohydrodynamic (MHD) equations in the regime of highly supersonic turbulence and on radiative transfer calculations. In some works, stochastic fields are used instead of the solution of the MHD equations, and in most studies the radiative transfer calculation is omitted in favor of density-weighted velocity profiles. The first large synthetic spectral maps of molecular transitions computed by solving the non-LTE radiative transfer through the density and velocity data cubes obtained as the numerical solution of the MHD equations were presented by Padoan et al. (1998), based on Juvela's radiative transfer code (Juvela 1997), and were used in a number of works (e.g., Padoan et al. 1999, 2000, 2001a). Another new radiative transfer code has also been used more recently to generate synthetic spectral maps from MHD simulations (Ossenkopf 2002).

A number of statistical methods have been proposed to compare numerical models of turbulence with large spectral maps of molecular clouds (see, e.g., Scalo 1984; Kleiner \& Dickman 1985, 1987; Stutzki \& Gusten 1990; Gill \& Henriksen 1990; Houlahan \& Scalo 1992; Hobson 1992; Langer, Wilson, \& Anderson 1993; Williams, De Geus, \& Blitz 1994; Miesch \& Bally 1994; Miesch \& Scalo 1995; Lis et al. 1996; Blitz \& Williams 1997; Heyer \& Schloerb 1997; Stutzki et al. 1998; Miesch, Scalo, \& Bally 1999; Falgarone et al. 1994; Padoan et al. 1999; Mac Low \& Ossenkopf 2000; Bensch, Stutzki, \& Ossenkopf 2001).

In this work we apply the spectral correlation function (SCF) method, proposed by Rosolowsky et al. (1999) and further developed in Padoan, Rosolowsky, \& Goodman (2001b), to a number of observational and synthetic spectral 
maps. We show that the slope and normalization of the SCF of observational maps correlate with the spectral line width. Theoretical models of molecular clouds should therefore yield synthetic spectral maps reproducing such correlations, but not all of them can.

In the next section we briefly define the SCF, and in $\S 3$ we present the observational data used in this work. The computation of the theoretical models and synthetic spectral maps is presented in $\S 4$. Results from numerical models are compared with the observational data in $\S 5$ and are discussed in $\S 6$. Conclusions are drawn in $\S 7$.

\section{THE SCF METHOD}

The SCF measures the spatial correlation of spectral line profiles within a spectral map. It is sensitive to the properties of both the gas mass distribution and the gas velocity field (Rosolowsky et al. 1999; Padoan et al. 2001a, 2001b; Ballesteros-Paredes, Vázquez-Semadeni, \& Goodman 2002).

Let $T(\boldsymbol{x}, v)$ be the antenna temperature as a function of velocity channel $v$ at map position $\boldsymbol{x}$. The SCF for spectra with spatial separation $\ell$ is

$$
S_{0}(\ell)=\left\langle\frac{S_{0}(\boldsymbol{x}, \ell)}{S_{0, N}(\boldsymbol{x})}\right\rangle_{\boldsymbol{x}},
$$

where the average is computed over all map positions $\boldsymbol{x}$. $S_{0}(\boldsymbol{x}, \ell)$ is the SCF uncorrected for the effects of noise,

$$
S_{0}(\boldsymbol{x}, \ell)=\left\langle 1-\sqrt{\frac{\Sigma_{v}[T(\boldsymbol{x}, v)-T(\boldsymbol{x}+\Delta \boldsymbol{x}, v)]^{2}}{\Sigma_{v} T(\boldsymbol{x}, v)^{2}+\Sigma_{v} T(\boldsymbol{x}+\Delta \boldsymbol{x}, v)^{2}}}\right\rangle_{\Delta \boldsymbol{x}},
$$

where the average is limited to separation vectors $\Delta \boldsymbol{x}$ with $|\Delta \boldsymbol{x}|=\ell$ and $S_{0, N}(\boldsymbol{x})$ is the SCF due to noise alone,

$$
S_{0, N}(\boldsymbol{x})=1-\frac{1}{Q(\boldsymbol{x})},
$$

and $Q(x)$ is the "spectrum quality" (see discussion in Padoan et al. 2001b). $Q(\boldsymbol{x})$ is defined as the ratio of the rms signal within a velocity window $W$ and the rms noise, $N$ (over all velocity channels),

$$
Q(\boldsymbol{x})=\frac{1}{N} \sqrt{\frac{\sum_{v} T(\boldsymbol{x}, v)^{2} d v}{W}},
$$

where $d v$ is the width of the velocity channels.

In the present work we compute the SCF of both observational and synthetic spectral maps, obtained by computing the radiative transfer through the three-dimensional density and velocity fields of numerical simulations of supersonic MHD turbulence. The result is typically a power law for $S_{0}(\ell)$ that extends up to a separation $\ell$ comparable to the map size, reflecting the self-similarity of supersonic turbulence (Padoan et al. 2001b). The power-law behavior is sometimes interrupted at an intermediate scale, possibly suggesting the presence of a physical mechanism limiting the inertial range of turbulence. An example of an SCF that defines an intermediate scale is the SCF of the H I survey of the Large Magellanic Cloud (LMC) by Kim et al. (1998, 1999). Padoan et al. (2001a) have recently been able to map the gas disk thickness of the LMC, assuming that it is related to the intermediate scale defined by the break in the SCF power law.

\section{THE OBSERVATIONAL DATA}

The absolute value of $S_{0}(\ell)$ at any $\ell$ and the slope of the $S_{0}(\ell)$ power law for any given region depend on which molecular tracer is used (Padoan et al. 2001b). Transitions probing higher gas density produce more fragmented integrated intensity maps than transitions probing lower gas density, and their SCF is therefore steeper. In order to compare the SCF of observational and synthetic maps, it is therefore important to solve the radiative transfer through the model density and velocity fields accurately for the same molecular transition that is observed.

In this work our aim is to compute the SCF of observational data in order to provide constraints for theoretical models. The best constraints come from computing the SCF of spectral maps of a specific molecular transition over a large range of line width and linear size. Observationally, smallscale and narrow-line width objects are usually mapped out with high-density tracers, while larger objects are instead usually probed with lower density tracers. ${ }^{13} \mathrm{CO}$ provides a good compromise, since it is the only molecule for which very large maps containing thousands of spectra have been obtained with a significant range of resolution. In this work we have therefore chosen to use observational and synthetic maps of the $J=1-0$ line of ${ }^{13} \mathrm{CO}$.

We have used $11{ }^{13} \mathrm{CO}$ maps. For each map, we have listed in Table 1 the approximate size, distance, rms velocity computed as the standard deviation of the line profile averaged over the whole map, telescope beam size, spatial sampling, width of the velocity channels, and spectral quality defined in the previous section. Smaller maps have been obtained from portions of the maps of the Taurus, Perseus, and Rosette molecular cloud complexes, and the SCF has been computed for each of them. The positions of these smaller maps within the molecular cloud complexes are shown in Figures 1 and 2. They have been called T1-T7 in Taurus, P1-P5 in Perseus, R1B in the Rosette molecular cloud map by Blitz \& Stark (1986) and R1 and R2 in the Rosette molecular cloud map by M. H. Heyer et al. (2003, in preparation). ${ }^{4}$

The SCF of each map has been approximated with a power law, over the range of spatial separations where a power-law fit is relevant. For each power-law fit, we compute its slope, $\alpha$, and its absolute value at $1 \mathrm{pc}, S_{0}(1 \mathrm{pc})$ :

$$
S_{0}(\ell)=S_{0}(1 \mathrm{pc})\left(\frac{\ell}{1 \mathrm{pc}}\right)^{-\alpha} .
$$

The values of $\alpha, S_{0}\left(1 \mathrm{pc}\right.$ ), and $\sigma_{v}$ (the line-of-sight rms velocity) and the Galactic coordinates of the center of each map are given in Table 2. The SCF of maps of molecular cloud complexes and some smaller regions are shown in Figure 3.

\section{MHD SIMULATIONS AND SYNTHETIC SPECTRAL MAPS}

We solve the compressible MHD equations in a staggered mesh of $128^{3}$ computational cells, with volume-centered

\footnotetext{
${ }^{4}$ More conventional designations for some of the subregions are given in Table 2.
} 
TABLE 1

Main Parameters of the ObServed Spectral Maps

\begin{tabular}{|c|c|c|c|c|c|c|c|c|}
\hline $\begin{array}{l}\text { MC } \\
\text { (1) }\end{array}$ & $\begin{array}{c}L \\
(\mathrm{pc}) \\
(2)\end{array}$ & $\begin{array}{c}D \\
(\mathrm{Kpc}) \\
(3)\end{array}$ & $\begin{array}{c}\sigma_{v} \\
\left(\mathrm{~km} \mathrm{~s}^{-1}\right) \\
(4)\end{array}$ & $\begin{array}{c}\text { Beam } \\
(\mathrm{pc}) \\
(5)\end{array}$ & $\begin{array}{l}d x \\
(\mathrm{pc}) \\
(6)\end{array}$ & $\begin{array}{c}d v \\
\left(\mathrm{~km} \mathrm{~s}^{-1}\right) \\
(7)\end{array}$ & $\begin{array}{c}\langle Q\rangle \\
(8)\end{array}$ & $\begin{array}{l}\text { Reference } \\
\text { (9) }\end{array}$ \\
\hline Taurus ................. & 30 & 0.14 & 0.97 & 0.11 & 0.081 & 0.10 & 2.3 & Mizuno et al. 1995 \\
\hline Perseus.................. & 30 & 0.30 & 2.01 & 0.16 & 0.087 & 0.27 & 2.8 & Padoan et al. 1999 \\
\hline Rosette ............... & 45 & 1.60 & 2.45 & 0.84 & 0.70 & 0.68 & 2.1 & Blitz \& Stark 1986 \\
\hline Rosette .............. & 35 & 1.60 & 1.86 & 0.36 & 0.23 & 0.06 & 3.8 & M. H. Heyer et al. 2001, in preparation \\
\hline $\mathrm{L} 1524 \ldots \ldots \ldots \ldots \ldots \ldots$ & 1.5 & 0.14 & 0.79 & 0.032 & 0.015 & 0.10 & 5.2 & F. Bensch 2002, private communication \\
\hline Polaris.................. & 1.5 & 0.11 & 0.70 & 0.025 & 0.012 & 0.10 & 1.3 & Bensch et al. 2001 \\
\hline 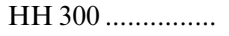 & 2 & 0.14 & 1.24 & 0.032 & 0.023 & 0.022 & 1.5 & Arce \& Goodman 2001 \\
\hline PV Ceph.............. & 3 & 0.50 & 0.98 & 0.114 & 0.083 & 0.022 & 1.8 & Arce \& Goodman 2001 \\
\hline Polaris................. & 0.3 & 0.11 & 0.53 & 0.012 & 0.004 & 0.052 & 6.3 & Falgarone et al. 1998 \\
\hline L1512 ................... & 0.3 & 0.15 & 0.20 & 0.016 & 0.005 & 0.052 & 11.7 & Falgarone et al. 1998 \\
\hline L134a..................... & 0.3 & 0.15 & 0.24 & 0.016 & 0.005 & 0.052 & 13.3 & Falgarone et al. 1998 \\
\hline
\end{tabular}

Note.-Col. (2): Approximate size. Col. (3): Distance. Col. (4): rms velocity over the whole map. Col. (5): Telescope beam. Col. (6): Spatial sampling. Col. (7): Velocity channel width. Col. (8): Average spectrum quality. Col. (9): Bibliographic reference.

mass density and thermal energy, face-centered velocity and magnetic field components, edge-centered electric currents and electric fields, and periodic boundary conditions. The code uses shock- and current sheet-capturing techniques to ensure that magnetic and viscous dissipation at the smallest
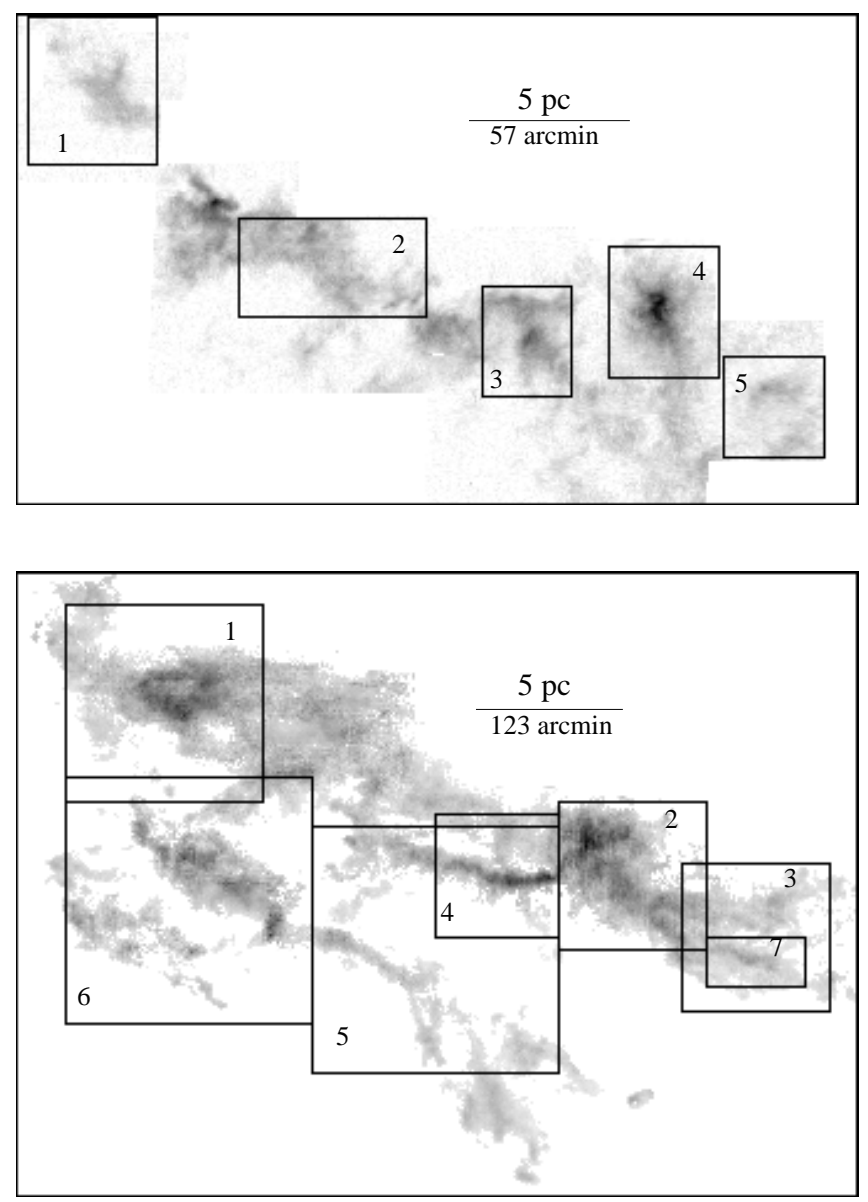

FIG. 1.-Top: Velocity-integrated intensity map of the Perseus molecular cloud complex in the $J=1-0$ transition of ${ }^{13} \mathrm{CO}$ (Padoan et al. 1999). Bottom: Same as the top panel, but for the Taurus molecular cloud complex (Mizuno et al. 1995). Smaller regions within the maps where the SCF has also been computed are highlighted. resolved scales provides the necessary dissipation paths for magnetic and kinetic energy. A more detailed presentation of the numerical method can be found elsewhere (Padoan \& Nordlund 1999).

For the purpose of the present work, we have computed numerical solutions of the MHD equations using an isothermal equation of state and a random driving force. In all experiments, the initial density is uniform, and the initial velocity is random. We generate the velocity field in Fourier space, and we give power, with a normal distribution, only
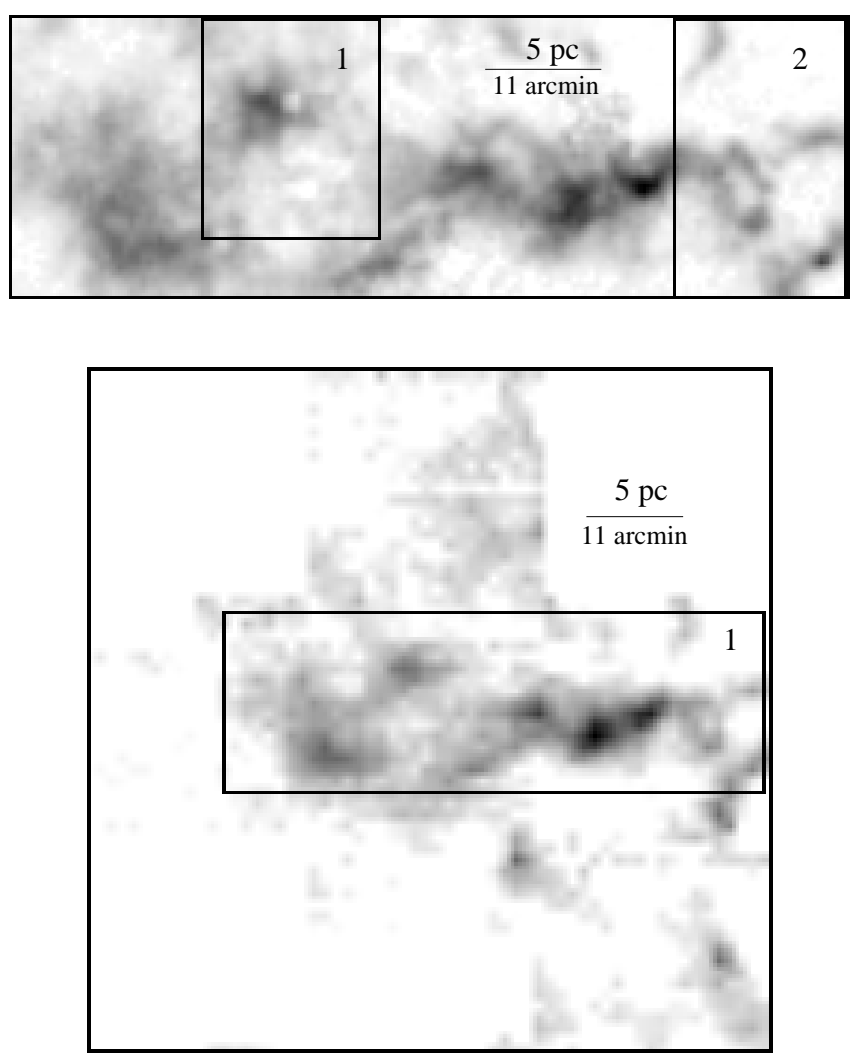

Fig. 2.- Same as in Fig. 1, but for the Rosette molecular cloud complex. The top panel is from M. H. Heyer et al. (2001, in preparation), and the bottom panel is from Blitz \& Stark (1986). 
TABLE 2

VALUES FROM OBSERVATIONAL MAPS

\begin{tabular}{|c|c|c|c|c|c|}
\hline $\begin{array}{l}\text { Region } \\
\text { (1) }\end{array}$ & $\begin{array}{c}\sigma_{v} \\
\left(\mathrm{~km} \mathrm{~s}^{-1}\right) \\
(2)\end{array}$ & $\begin{array}{l}\alpha \\
(3)\end{array}$ & $\begin{array}{c}S_{0}(1 \mathrm{pc}) \\
\quad(4)\end{array}$ & $\begin{array}{c}l \\
(\mathrm{deg}) \\
(5)\end{array}$ & $\begin{array}{c}b \\
\text { (deg) } \\
(6)\end{array}$ \\
\hline Taurus .......... & 0.97 & 0.24 & 0.47 & 170.8 & -16.2 \\
\hline $\mathrm{T} 1$ & 0.71 & 0.27 & 0.46 & 174.5 & -13.8 \\
\hline 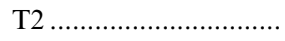 & 0.85 & 0.26 & 0.48 & 168.0 & -16.1 \\
\hline T3 .............................. & 0.53 & 0.25 & 0.46 & 166.3 & -16.8 \\
\hline $\mathrm{T} 4 \ldots$ & 0.96 & 0.32 & 0.41 & 169.8 & -16.1 \\
\hline T5 ... & 0.99 & 0.31 & 0.41 & 170.8 & -17.0 \\
\hline T6 ....... & 0.85 & 0.30 & 0.40 & 174.2 & -16.3 \\
\hline 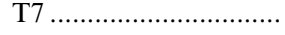 & 0.42 & 0.27 & 0.46 & 166.2 & -17.3 \\
\hline Perseus............................. & 2.01 & 0.32 & 0.42 & 160.0 & -19.3 \\
\hline 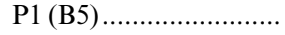 & 1.20 & 0.29 & 0.51 & 161.2 & -16.8 \\
\hline P2 & 1.33 & 0.30 & 0.44 & 160.7 & -18.8 \\
\hline P3 (B1). & 1.03 & 0.27 & 0.45 & 159.8 & -20.1 \\
\hline P4 (NGC 1333) ............. & 1.33 & 0.34 & 0.53 & 158.8 & -20.5 \\
\hline P5 (L1448) ................... & 1.53 & 0.29 & 0.51 & 158.6 & -21.6 \\
\hline Rosette (Bell Lab) ........ & 2.45 & 0.50 & 0.65 & 207.5 & -1.8 \\
\hline R1B & 2.18 & 0.42 & 0.60 & 207.3 & -1.8 \\
\hline Rosette (FCRAO) ....... & 1.86 & 0.39 & 0.52 & 207.3 & -1.8 \\
\hline $\mathrm{R} 1 \ldots$ & 1.40 & 0.38 & 0.45 & 207.5 & -1.8 \\
\hline $\mathrm{R} 2$. & 0.86 & 0.36 & 0.50 & 206.9 & -1.8 \\
\hline L1524... & 0.79 & 0.23 & 0.44 & 173.3 & -16.3 \\
\hline Polaris $($ FCRAO) ........ & 0.70 & 0.27 & 0.32 & 123.4 & 24.9 \\
\hline 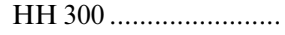 & 1.24 & 0.30 & 0.35 & 172.9 & -16.7 \\
\hline 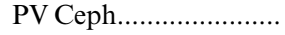 & 0.98 & 0.32 & 0.39 & 102.9 & 15.2 \\
\hline Polaris (IRAM) ............ & 0.54 & 0.27 & 0.26 & 123.7 & 24.9 \\
\hline L1512 & 0.20 & 0.18 & 0.39 & 171.8 & -5.2 \\
\hline L134a & 0.24 & 0.13 & 0.49 & 4.3 & 35.8 \\
\hline
\end{tabular}

Note. - Col. (2): Spectral line width averaged over the whole map, $\sigma_{v}$. Col. (3): Power-law slope of the SCF, $\alpha$. Col. (4): SCF normalization, $S_{0}(1 \mathrm{pc})$. Cols. (5) and (6): Galactic longitude, $l$, and galactic latitude, $b$, of the center of all the observed maps and selected regions within them.

to the Fourier components in the shell of wavenumbers $1 \leq k L / 2 \pi \leq 2$. We perform a Helmholtz decomposition and use only the solenoidal component of the initial velocity. However, a compressional component of the velocity field develops almost immediately because of the flow compressibility. The external driving force is generated on large scales in the same way as the velocity field. The initial magnetic field is uniform and is oriented parallel to the $z$-axis: $\boldsymbol{B}=B_{0} \hat{z}$.

Because of the limited numerical resolution, we have chosen not to model the collapse of turbulent density fluctuations. Self-gravity has therefore been neglected. We have recently started to compute turbulent self-gravitating flows with a numerical mesh of $500^{3}$ cells. Results of the analysis of these larger simulations, including self-gravity, will be presented in future works.

\subsection{Numerical Models}

We have run a number of MHD simulations in a $128^{3}$ computational mesh, with periodic boundary conditions. The simulations are intended to describe the turbulent dynamics in the interior of molecular clouds. The two most important numerical parameters in the models are the rms sonic and Alfvénic Mach numbers, $M_{S}$ and $M_{\mathrm{A}}$, respectively. The rms sonic Mach number is here defined as the ratio of the rms flow velocity and the speed of sound. The Alfvénic Mach number is defined as the ratio of the rms flow velocity and the Alfvén velocity, $v_{\mathrm{A}}=B /(4 \pi \rho)^{1 / 2}$, where $B$ is the volume-averaged magnetic field strength.

All the models used in this work have $M_{\mathrm{A}}=10$, except for model $\mathrm{E}$, which has $M_{\mathrm{A}}=1$, according to the suggestion that the dynamics of molecular clouds is essentially superAlfvénic (Padoan \& Nordlund 1999). Our numerical simulations conserve magnetic flux, and so the volume-averaged magnetic field is constant in time. As a consequence, the value of $M_{\mathrm{A}}$ as defined above also remains constant. However, the value of $B^{2}$ grows with time (until equilibrium is reached) because of compression and stretching of magnetic field lines (see Padoan \& Nordlund 1999). If we define the Alfvén velocity using the rms value of the magnetic field strength, instead of its volume average, then the typical Alfvénic Mach number in our super-Alfvénic runs is $M_{\mathrm{A}} \approx 2$, because of the formation of regions with a large value of magnetic field strength (mainly dense regions, as found in observations).

The sonic Mach number of observed turbulent motions in molecular clouds is $M_{S}>10$ on the scale of several parsecs, and it decreases toward smaller scales. The turbulent velocity becomes comparable to the speed of sound only on a very small scale, $\leq 0.1 \mathrm{pc}$. In order to study the effect of the sonic Mach number on the SCF, we have computed MHD models with different values of $M_{S}, M_{S}=10,5,2.5,1.25$, and 0.625 . Each model has been run for approximately six dynamical times (the dynamical time is here defined as the ratio of half the size of the computational box and the rms flow velocity), in order to achieve a statistically relaxed state, independent of the initial conditions.

The velocity and density fields from the final snapshot of each model have been used to compute ${ }^{13} \mathrm{CO} J=1-0$ spectra, solving the radiative transfer with a non-LTE Monte Carlo code ( $\S 4.2)$. While the MHD calculations are independent of the physical value of the average gas density, the size of the computational mesh (or the column density), and the kinetic temperature, these physical parameters are necessary inputs for the radiative transfer calculations.

The models are scaled to physical units assuming a value for (1) the kinetic temperature, $T_{K}$, that determines the physical unit of velocity (the numerical unit of velocity is the speed of sound), (2) the average gas density, $\langle n\rangle$, and (3) the size of the computational box, $L_{0}$. For all models, we have assumed $T_{K}=10 \mathrm{~K}$, typical of molecular clouds. The dependence of observed average gas density and cloud size on the observed rms turbulent velocity (or sonic Mach number, assuming a constant value of $T_{K}$ ) is well approximated by empirical Larson-type relations (Larson 1981). However, the size-velocity relation has a large intrinsic scatter (Falgarone, Puget, \& Pérault 1992), and both the sizevelocity and density-size relations have been criticized by several authors (Loren 1989; Kegel 1989; Scalo 1990; Issa et al. 1990; Adler \& Roberts 1992; Vázquez-Semadeni, Ballesteros-Paredes, \& Rodríguez 1997; Ostriker et al. 2001; Ballesteros-Paredes \& Mac Low 2002). For these reasons, we scale the MHD models in four different ways. These four sets of models are all based on the same five MHD turbulence models and differ from each other only in the way they are rescaled to physical units when computing the radiative transfer. Models A1-A5 and B1-B5 have all the same value of the average density, $\langle n\rangle=300 \mathrm{~cm}^{-3}$. Models A1-A5 have all the same size $L_{0}=5 \mathrm{pc}$ and column density $N_{\text {col }}=$ $4.5 \times 10^{21} \mathrm{~cm}^{-2}$; models $\mathrm{B} 1-\mathrm{B} 5$ have $L_{0}=20 \mathrm{pc}$ and $N_{\text {col }}=1.8 \times 10^{22} \mathrm{~cm}^{-2}$. Models A1R-A5R and B1R-B5R 

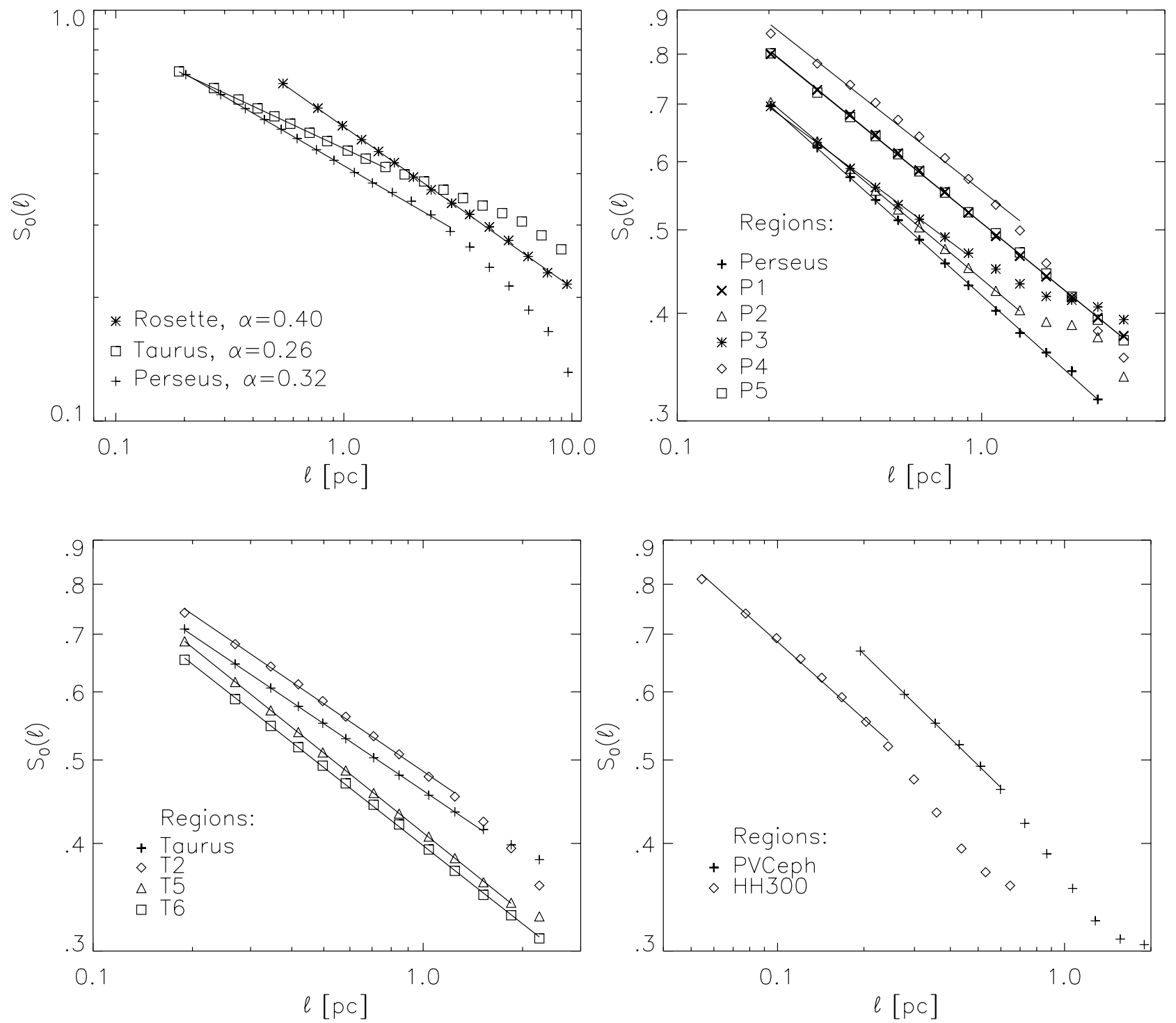

FIG. 3.-Top left: SCF averaged over the entire map of the Perseus, Rosette, and Taurus molecular cloud complexes. Solid lines show the least-squares fits to the power-law sections of the SCF. The exponents $\alpha$ of the power-law fits are also given in the figure. Top right: SCF of the whole map of the Perseus molecular cloud complex and of smaller regions within the same map. Bottom left: SCF of the Taurus molecular cloud complex and of smaller regions within the same map. Bottom right: SCF of PV Ceph and HH 300.

are rescaled using the Larson-type relations:

$$
M_{S}=M_{S, 1 \mathrm{pc}}\left(\frac{L}{1 \mathrm{pc}}\right)^{0.5}
$$

where a temperature $T_{K}=10 \mathrm{~K}$ is assumed, and

$$
\langle n\rangle=n_{1 \mathrm{pc}}\left(\frac{L}{1 \mathrm{pc}}\right)^{-1}
$$

which is equivalent to a constant mean surface density. Models A1R-A5R have the same column density as models A1-A5, that is, $n_{1 \mathrm{pc}}=1.5 \times 10^{3} \mathrm{~cm}^{-3}$ in equation (7); they also have sizes $L_{0}=10,2.5,0.625,0.156$, and $0.039 \mathrm{pc}$, respectively, which implies $M_{S, 1 \mathrm{pc}}=3.16$ in equation (6). Models B1R-B2R have the same column density as models $\mathrm{B} 1-\mathrm{B} 5$, that is, $n_{1 \mathrm{pc}}=6.0 \times 10^{3} \mathrm{~cm}^{-3}$ in equation (7); they have sizes $L_{0}=20,5,1.25,0.31$, and $0.078 \mathrm{pc}$, respectively, which implies $M_{S, 1 \mathrm{pc}}=2.23$ in equation (6). Finally, the equipartition model (model E) has been computed only for one value of the rms sonic Mach number, $M_{S}=10$. It is rescaled to the Larson-type relations only once, for a size of $10 \mathrm{pc}$ and a column density of $N_{\mathrm{col}}=4.5 \times 10^{21} \mathrm{~cm}^{-2}$. For this model, we have computed spectral maps along five different directions, three orthogonal to the faces of the numerical mesh, as in the other experiments, and two along diagonal directions, at an angle of 54.7 with the average magnetic field ( $z$-axis).

Maps from diagonal directions sample lines of sight of different length at different map positions (longer at the central position than near the corners). However, the number of computational cells along each line of sight is on the average even larger than in maps from orthogonal directions, since a diagonal line of sight often cuts through the computational cells away from their center (close to their corners). Furthermore, the maps are computed only for a region of size equivalent to that of maps from orthogonal directions $(90 \times 90$ cells), eliminating the corners of the computational 
mesh. As a result, only a few percent of the spectra are generated from lines of sight sampling fewer than 50 computational cells. A fraction of the lines of sight close to the map edges are nevertheless shorter than the energy injection scale (approximately half the size of the computational mesh). This may introduce a bias toward smaller line width, since velocity differences are expected to grow with increasing distances. This bias, or its effect on the SCF, should be small, since our results seem to vary smoothly as a function of the angle between the line of sight and the direction of the average magnetic field.

Models A4, A5, B3, B4, and B5 have velocity dispersion significantly smaller than found observationally at the scale of 5 pc. They are not used here to test the validity of models with such low velocity dispersion, but rather to test the ability of the SCF method to rule them out as poor description of molecular cloud turbulence.

In order to test the ability of the SCF to rule out unphysical models, we have also computed two stochastic models, S2 and S4. In both models the density field is a random field with a lognormal probability distribution function and a power-law power spectrum with a power-law exponent equal to -1 (the approximate value found in our MHD models). The velocity field is generated as a Gaussian field, also with power-law power spectrum. The power-law exponent of the velocity field power spectrum is -2 (close to the actual value in the MHD models) in model $\mathrm{S} 2$ and -4 in model S4. For the purpose of computing the radiative transfer and the synthetic spectral maps, both models have been scaled to a physical size $L_{0}=20$ pc and a column density $N_{\text {col }}=4.5 \times 10^{21} \mathrm{~cm}^{-2}$.

These two stochastic models are unphysical in the sense that they are not solutions of the fluid equations. Statistical properties such as the power spectrum and the probability density function of density and velocity may be similar to those of flows obtained by solving the fluid equations, but their phase correlations are unphysical. This is in part illustrated by the fact that these stochastic models look clumpy, rather than filamentary as real clouds and MHD models do. Furthermore, their velocity and density fields cannot be self-consistent because they are computed independently of each other. It is shown below that the SCF method can indeed rule out these unphysical models.

The sonic rms Mach number, $M_{S}$, the average gas density, $\langle n\rangle$, and the physical size, $L_{0}$, of the different models used for the radiative transfer computations are given in Table 3.

\subsection{Radiative Transfer and Synthetic Spectral Maps}

The radiative transfer calculations were carried out with a Monte Carlo program, which is a generalization of the onedimensional Monte Carlo method (Bernes 1979) into three dimensions. The model cloud is divided into small, cubic cells in which physical properties are assumed to be constant. The discretization allows the inclusion of arbitrary kinetic temperature and molecular abundance variations. However, in the present calculations, the temperature and relative abundances are kept constant. The $2.73 \mathrm{~K}$ cosmic background is used as the external radiation field. There are important differences between our program and the normal Monte Carlo method, and some principles of the implementation are given below. A detailed description is given elsewhere (Juvela 1997).
In the basic Monte Carlo method the radiation field is simulated with photon packages, each representing a number of real photons. The packages are created at random velocities, at random locations, and sent toward random directions. Each package is followed through the cloud, and interactions between photons and molecules are counted. Later this information is used to solve new estimates for the level populations of the molecules.

In our method the radiative transfer is simulated along random lines going through the cloud. Initially, as a photon package enters the cloud, it contains only background photons. As the package goes through a cell in the cloud, some photons emitted by this cell are added to the package, and, in particular, the number of photons absorbed within the emitting cell is calculated explicitly. This becomes important when cells are optically thick and, compared with normal Monte Carlo simulation, ensures more accurate estimation of the energy transfer between cells. In our program each simulated photon package represents the intensity of all simulated transitions and Doppler shifts at the same time. The number of individual photon packages is correspondingly smaller, and in the present case we use 240,000 photon packages per iteration. The lines are divided into 70 fixed velocity channels. There is no noise associated with random sampling of Doppler shifts. The simulated velocity range was adjusted according to the velocity range found in the model clouds. The channels are narrow compared to the total line widths and smaller than or equal to the smallest intrinsic line widths in the cells. The velocity discretization is therefore not expected to affect the results of the calculations.

The density and velocity fields from the MHD simulations are sampled on a numerical mesh of $128^{3}$ cells. To speed up the radiative transfer calculations, the density and velocity fields were rebinned into a mesh of $90^{3}$ cells by linear interpolation. The velocity dispersion between neighboring cells in the original $128^{3}$ data cube was used to approximate the turbulent line width within each cell of the new $90^{3}$ data cube. This velocity dispersion should apply to a scale slightly larger than the size of the cells in the $90^{3}$ mesh. However, this is approximately compensated by the fact that numerical dissipation in the MHD simulations decreases significantly the velocity dispersion on very small scale, below the actual turbulent inertial range value at that scale.

On each iteration new level populations are solved from the equilibrium equations, and iterations are stopped when the relative change is below $\sim 2.0 \times 10^{-4}$ in all cells. Only the six lowest levels were tested for convergence. The relative changes tend to be largest on the upper levels, where the level populations become very small, and, on the average, the convergence of the relevant first energy levels is much better than the quoted limit. The total number of energy levels included in the calculations was nine, a number clearly sufficient in case of excitation temperatures below $10 \mathrm{~K}$. The collisional coefficients were taken from Flower \& Launay (1985) and Green \& Thaddeus (1976).

The final level populations were used to calculate maps of $90 \times 90$ spectra toward three directions perpendicular to the faces of the MHD data cube. For the equipartition model E, spectra were calculated also along two diagonal directions. In these cases the maps of $90 \times 90$ spectra do not extend over the whole projected cloud area. Each spectrum corresponds to the intensity calculated along one line of sight 
TABLE 3

Values From Theoretical Models

\begin{tabular}{|c|c|c|c|c|c|c|c|c|c|c|c|}
\hline \multirow[b]{2}{*}{$\begin{array}{l}\text { ModEL } \\
\text { (1) }\end{array}$} & \multirow[b]{2}{*}{$\begin{array}{c}M_{S} \\
(2)\end{array}$} & \multirow[b]{2}{*}{$\begin{array}{c}L_{0} \\
(\mathrm{pc}) \\
(3)\end{array}$} & \multicolumn{3}{|c|}{$x$} & \multicolumn{3}{|c|}{$y$} & \multicolumn{3}{|c|}{$z$} \\
\hline & & & $\begin{array}{c}\sigma_{v} \\
\left(\mathrm{~km} \mathrm{~s}^{-1}\right) \\
(4)\end{array}$ & $\begin{array}{c}\alpha \\
(5)\end{array}$ & $\begin{array}{c}S_{0}(1 \mathrm{pc}) \\
\quad(6)\end{array}$ & $\begin{array}{c}\sigma_{v} \\
\left(\mathrm{~km} \mathrm{~s}^{-1}\right) \\
(7)\end{array}$ & $\begin{array}{c}\alpha \\
(8)\end{array}$ & $\begin{array}{c}S_{0}(1 \mathrm{pc}) \\
\quad(9)\end{array}$ & $\begin{array}{c}\sigma_{v} \\
\left(\mathrm{~km} \mathrm{~s}^{-1}\right) \\
(10)\end{array}$ & $\begin{array}{c}\alpha \\
(11)\end{array}$ & $\begin{array}{c}S_{0}(1 \mathrm{pc}) \\
\quad(12)\end{array}$ \\
\hline \multicolumn{12}{|c|}{ MHD Models $\left(\langle n\rangle=300 \mathrm{~cm}^{-3}\right)$} \\
\hline $\mathrm{A} 1 \ldots \ldots \ldots$ & 10.0 & 5 & 1.13 & 0.30 & 0.37 & 1.36 & 0.32 & 0.34 & 1.24 & 0.35 & 0.31 \\
\hline $\mathrm{A} 22 \ldots \ldots \ldots$ & 5.0 & 5 & 0.56 & 0.25 & 0.46 & 0.71 & 0.30 & 0.39 & 0.67 & 0.28 & 0.42 \\
\hline $\mathrm{A} 33 \ldots \ldots \ldots$ & 2.5 & 5 & 0.30 & 0.19 & 0.55 & 0.38 & 0.24 & 0.48 & 0.36 & 0.21 & 0.52 \\
\hline $\mathrm{A} 4{ }_{\ldots} \ldots \ldots \ldots$ & 1.2 & 5 & 0.17 & 0.13 & 0.66 & 0.17 & 0.15 & 0.64 & 0.18 & 0.16 & 0.61 \\
\hline A $5 \ldots \ldots \ldots$ & 0.6 & 5 & 0.12 & 0.13 & 0.68 & 0.11 & 0.10 & 0.71 & 0.12 & 0.14 & 0.66 \\
\hline B1 ............. & 10.0 & 20 & 1.21 & 0.26 & 0.64 & 1.42 & 0.27 & 0.61 & 1.33 & 0.27 & 0.59 \\
\hline B2 ........... & 5.0 & 20 & 0.61 & 0.22 & 0.70 & 0.77 & 0.26 & 0.65 & 0.72 & 0.24 & 0.67 \\
\hline B3 .......... & 2.5 & 20 & 0.33 & 0.18 & 0.73 & 0.41 & 0.23 & 0.68 & 0.39 & 0.20 & 0.71 \\
\hline B4 ............ & 1.2 & 20 & 0.18 & 0.14 & 0.77 & 0.19 & 0.15 & 0.77 & 0.21 & 0.17 & 0.75 \\
\hline $\mathrm{B} 5 \ldots \ldots \ldots$ & 0.6 & 20 & 0.13 & 0.13 & 0.79 & 0.13 & 0.11 & 0.81 & 0.14 & 0.14 & 0.78 \\
\hline \multicolumn{12}{|c|}{ Rescaled MHD Models $\left(\langle n\rangle=6 \times 10^{3} \mathrm{~cm}^{-3} L_{0, \mathrm{pc}}^{-1}\right)$} \\
\hline A1R ....... & 10.0 & 10 & 1.13 & 0.30 & 0.44 & 1.36 & 0.32 & 0.42 & 1.23 & 0.35 & 0.39 \\
\hline A2R ...... & 5.0 & 2.5 & 0.56 & 0.23 & 0.41 & 0.72 & 0.30 & 0.32 & 0.66 & 0.26 & 0.37 \\
\hline A3R ....... & 2.5 & 0.62 & 0.30 & 0.18 & 0.41 & 0.37 & 0.21 & 0.34 & 0.35 & 0.20 & 0.37 \\
\hline A4R ...... & 1.2 & 0.16 & 0.16 & 0.12 & 0.45 & 0.15 & 0.13 & 0.42 & 0.17 & 0.15 & 0.38 \\
\hline A5R ...... & 0.6 & 0.04 & 0.10 & 0.11 & 0.41 & 0.10 & 0.08 & 0.49 & 0.11 & 0.12 & 0.39 \\
\hline B1R ....... & 10.0 & 20 & 1.21 & 0.26 & 0.64 & 1.42 & 0.27 & 0.61 & 1.33 & 0.27 & 0.59 \\
\hline B2R ...... & 5.0 & 5 & 0.60 & 0.22 & 0.55 & 0.76 & 0.23 & 0.49 & 0.69 & 0.20 & 0.53 \\
\hline B3R ....... & 2.5 & 1.25 & 0.33 & 0.15 & 0.52 & 0.40 & 0.18 & 0.45 & 0.37 & 0.16 & 0.49 \\
\hline B4R ....... & 1.2 & 0.31 & 0.18 & 0.12 & 0.50 & 0.18 & 0.13 & 0.47 & 0.19 & 0.14 & 0.44 \\
\hline $\mathrm{B} 5 \mathrm{R} \ldots \ldots$ & 0.6 & 0.08 & 0.12 & 0.10 & 0.46 & 0.12 & 0.09 & 0.52 & 0.13 & 0.12 & 0.42 \\
\hline \multicolumn{12}{|c|}{ Equipartition MHD Model } \\
\hline $\mathrm{E} \ldots \ldots \ldots \ldots$ & 10.0 & 5 & 0.73 & 0.21 & 0.46 & 0.81 & 0.25 & 0.43 & 1.90 & 0.49 & 0.22 \\
\hline \multicolumn{12}{|c|}{ Stochastic Models } \\
\hline $\mathrm{S} 2 \ldots \ldots \ldots$ & 10.0 & 20 & 1.37 & 0.15 & 0.52 & 1.29 & 0.15 & 0.53 & 1.31 & 0.14 & 0.53 \\
\hline S4 ........... & 10.0 & 20 & 1.23 & 0.16 & 0.47 & 1.17 & 0.15 & 0.49 & 1.17 & 0.17 & 0.46 \\
\hline
\end{tabular}

Note-Columns (1)-(3) give the model name, rms sonic Mach number of the flow, and physical size of the computational mesh. Columns (4)-(6), (7)-(9), and (10)-(12) give the line-of-sight velocity dispersion, SCF slope, and SCF normalization, repeated for the three orthogonal directions for which synthetic spectral maps have been computed in each model. Values for the diagonal directions of model $\mathrm{E}$ are not given (they are within the ranges of values covered by the other three directions parallel and perpendicular to the mean magnetic field).

(spectra are not convolved with a larger beam). The spectra contain 60 velocity channels, as in the Monte Carlo simulation. The results were compared with spectra calculated assuming LTE conditions. The comparison showed that for typical physical conditions found in molecular clouds, the LTE assumption would be unsuitable (Padoan et al. 2000).

\section{OBSERVATIONAL CORRELATIONS AND THEORETICAL MODELS}

Most observational and theoretical spectral maps yield an SCF that can be approximated by a single power law within a range of spatial separations, often spanning over an order of magnitude. From each power-law fit we compute its slope, $\alpha$, and its value at $1 \mathrm{pc}, S_{0}(1 \mathrm{pc})$, defined as in equation (5). We also compute the value of the velocity dispersion, $\sigma_{v}$, from each map, measured as the standard deviation of the ${ }^{13} \mathrm{CO} J=1-0$ spectrum averaged over the entire map. The values of $\alpha, S_{0}(1 \mathrm{pc})$, and $\sigma_{v}$ computed from the observational maps are given in Table 2, while the values of the same quantities from the theoretical models are given in Table 3.

Every model provides three sets of values, because spectral maps have been computed using three orthogonal directions for the line of sight. Each group of three sets of values can be interpreted as the same model cloud being "observed" from different directions or as three different model clouds with comparable rms velocity. In the equipartition model, E, the rms velocities inferred from different directions are very different from each other, the highest rms velocity being found in the direction parallel to the mean magnetic field (along the $z$-axis) and the lowest in the directions perpendicular to the magnetic field. For this model, we have also computed spectral maps from two more lines of sight, corresponding to diagonal directions across the computational box.

Figure 4 shows the SCF of the model A1R, with the line of sight parallel to the direction of the mean magnetic field. The SCF of the equipartition model $\mathrm{E}$ is also shown for four lines of sight, two diagonal, one parallel to the direction of the mean magnetic field, and one perpendicular to it to it. 


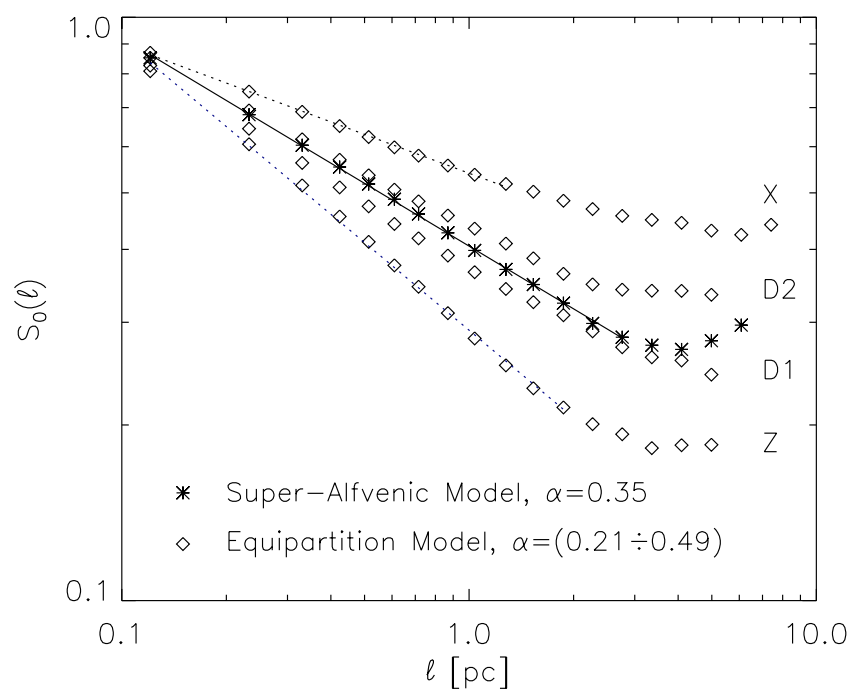

FIG. 4. - SCF computed from MHD models. Asterisks show the superAlfvénic model A1R in the $z$-direction (parallel to the mean magnetic field). The diamonds show the equipartition model in the $x$ - and $z$-directions (perpendicular and parallel to the average magnetic field direction, respectively) and along two diagonal directions ( $D 1$ and $D 2)$. The slope of the SCF increases with increasing rms velocity. The SCF is therefore weakly dependent on the direction of the line of sight for the super-Alfvénic model, while it is much steeper in the direction parallel to the magnetic field (larger rms velocity) than in the perpendicular direction in the equipartition model.

The figure shows that the SCF of model E is very sensitive to the line of sight because of the large variations of the rms velocity in different directions relative to the mean magnetic field.

The velocity dispersion relative to the speed of sound, or the value of the sonic Mach number, is the most important physical parameter characterizing the nature of the turbulence. We therefore study the dependence of the SCF on the turbulent velocity dispersion (or the rms sonic Mach number, $M_{S}$ ) and propose to use this dependence to test theoretical models against the observational data.

In Figure 5 the slope of the SCF is plotted against the line-of-sight velocity dispersion. The top panels show the models of constant size and constant average gas density (models A1-A5 [left] and B1-B5 [right]); the bottom panels show the models rescaled according to Larson-type relations (models A1R-A5R [left] and B1R-B5R [right]). The observations indicate a strong correlation between $\alpha$ and $\sigma_{v}$, over an order of magnitude in $\sigma_{v}$. A least-squares fit to the observational data gives

$$
\alpha_{\text {obs }}=0.30 \sigma_{v, \text { obs }}^{0.37 \pm 0.07},
$$

and for the supersonic and super-Alfvénic models rescaled with Larson-type relations (A1R-A5R),

$$
\alpha_{\mathrm{MHD}}=0.31 \sigma_{v, \mathrm{MHD}}^{0.47 \pm 0.04},
$$

consistent with the observational result (the uncertainty in the exponent is the standard deviation from the leastsquares fit). The corresponding models not scaled with the Larson-type relations (A1-A5) are also indistinguishable from the observational result (see Fig. 5, top left-hand panel). Models of type B (right-hand panels of Fig. 5) instead have values of $\alpha$ significantly smaller than the average ones from the observational data. The stochastic models
S2 and S4 are indistinguishable from each other; they are also totally inconsistent with the empirical $\alpha-\sigma_{v}$ relation, which allows them to be ruled out as invalid by the SCF method. Finally, the equipartition model E provides values that are consistent with the observations and comparable to the super-Alfvénic models, apart from a larger scatter of values between different lines of sight.

We interpret the increase of $\alpha$ with $\sigma_{v}$ as a consequence of the increasing compressibility of the turbulent flow ( $\sigma_{v}$ is roughly proportional to the rms sonic Mach number of the flow because the temperature in all the models is $T_{K}=10 \mathrm{~K}$ and approximately the same in the observed regions). The value of $\alpha$ is in general found to grow with increasing density contrast, probably because of the increasing concentration of the mass along the line of sight around one or few dense cores, which helps decorrelating the spectra from each other.

The value of $S_{0}(1 \mathrm{pc})$ is plotted against $\sigma_{v}$ in Figure 6 . The top panels show the models of constant size and the bottom panels the models scaled with the Larson-type relations, as in Figure 5. The values of $S_{0}(1 \mathrm{pc})$ and $\sigma_{v}$ from the observational maps are weakly correlated, with $S_{0}(1 \mathrm{pc})$ slightly increasing with increasing $\sigma_{v}$ :

$$
S_{0, \mathrm{obs}}(1 \mathrm{pc})=0.45 \sigma_{v, \mathrm{obs}}^{0.13 \pm 0.08} .
$$

A tight correlation is instead found in the models of constant size (top panels of Fig. 6), with $S_{0}(1 \mathrm{pc})$ decreasing with increasing $\sigma_{v}$. This inconsistency between the models and the observations is most likely due to the fact that molecular clouds of 5-20 pc of size (as assumed by these models) are never found with a velocity dispersion as low as assumed in models A4 and A5 and B3, B4, and B5. The bottom panels of Figure 6 show that the inconsistency is, in fact, mostly resolved as soon as the model sizes are scaled according to the Larson-type relation. For models A1RA5R, we obtain

$$
S_{0, \mathrm{MHD}}(1 \mathrm{pc})=0.39 \sigma_{v, \mathrm{MHD}}^{-0.03 \pm 0.05} .
$$

If models with $\sigma_{v}<0.2 \mathrm{~km} \mathrm{~s}^{-1}$ were not included (justified by the absence of such low velocity dispersions in the observational sample), the slope of the least-squares fit would be $0.07 \pm 0.04$, fully consistent with the observations. The equipartition model yields values of $S_{0}(1 \mathrm{pc})$ and $\sigma_{v}$ consistent with the observations in all directions but the one parallel to the mean magnetic field. It could be concluded that either none of the observed objects have a significant component of the magnetic field along the line of sight or all of them have a magnetic field weaker than predicted by the equipartition model, consistent with the super-Alfvénic models.

In Figure 7 we have plotted observations and models on the $\alpha-S_{0}(1 \mathrm{pc})$ plane. The constant-size models are again inconsistent with the observations, as is expected since the observational maps span a large range of scales. When the models are scaled according to the Larson-type relations and the realistic average column density of $4.5 \times 10^{21} \mathrm{~cm}^{-2}$ (Myers \& Goodman 1988), the observed scatter in the $\alpha-$ $S_{0}(1 \mathrm{pc})$ plane is reproduced. The trend of the absolute value of $\alpha$ to increase with $S_{0}(1 \mathrm{pc})$ for large values of both of them is also reproduced, between models with rms Mach 5 and 10 (A2R and A1R, respectively); however, models with rms Mach of 20 or 30 would be necessary to fit the $\alpha$ and 

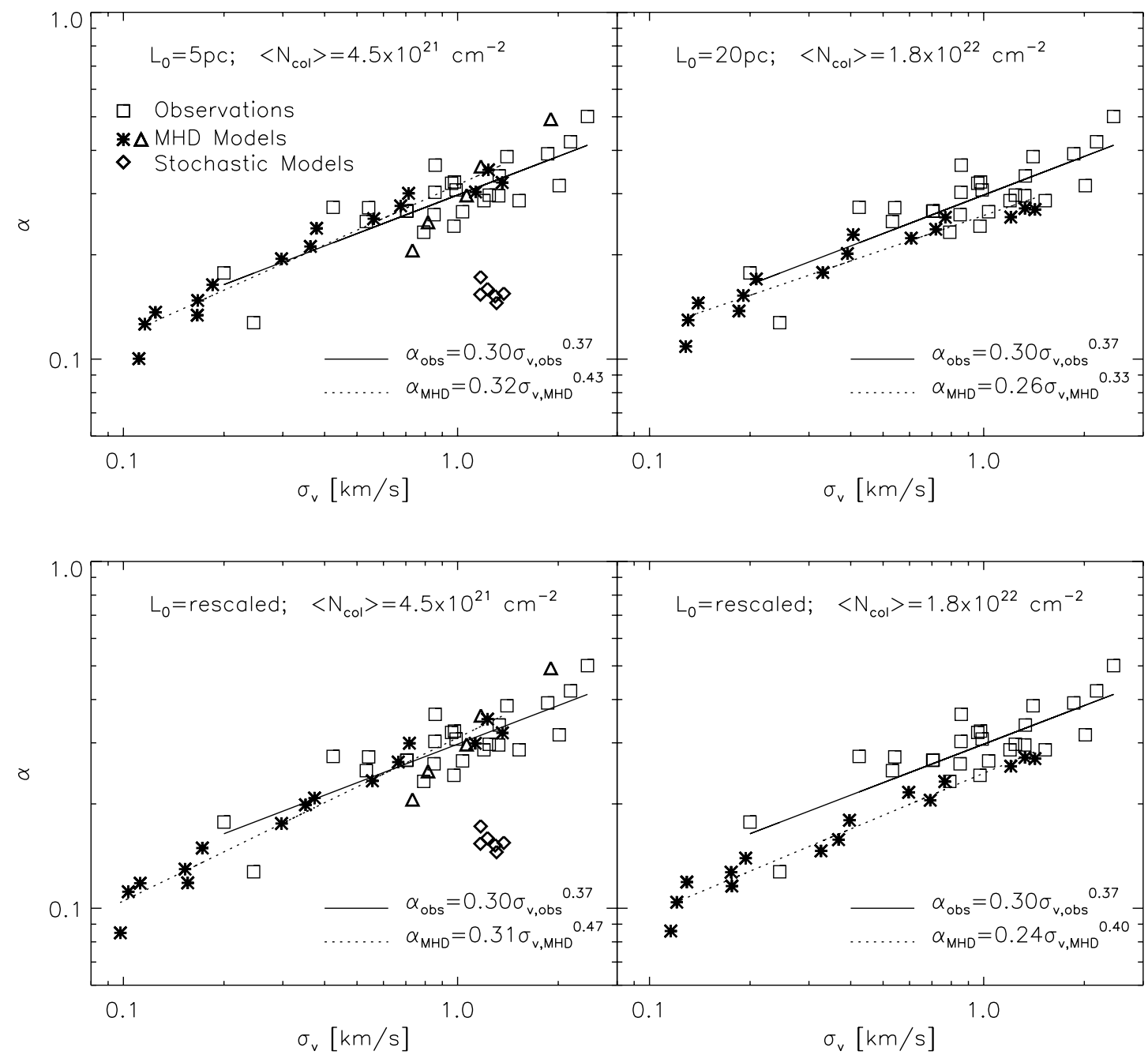

FIG. 5. - SCF slope vs. velocity dispersion. The top panels show the models of constant size and constant average gas density as asterisks (left: models A1A5; right: models B1-B5); the bottom panels show the models rescaled according to Larson-type relations as asterisks (left: models A1R-A5R; right: models B1R-B5R). Observational values are shown as squares, the equipartition model as triangles, and the stochastic models as diamonds.

$S_{0}(1 \mathrm{pc})$ values measured for the Rosette molecular cloud, which can be appropriately resolved only with a numerical resolution in excess of $256^{3}$ computational cells.

While the stochastic models S2 and S4 are only marginally inconsistent with the observations in this plot, the line of sight parallel to the direction of the mean magnetic field and one of the two diagonal lines of sight in the equipartition model $\mathrm{E}$ are again inconsistent with the observational data.

\section{DISCUSSION}

The SCF has been proposed as a statistical tool to test the validity of theoretical models describing the structure and dynamics of star-forming clouds (Rosolowsky et al. 1999). In a previous work we improved the SCF method by studying its dependence on spatial and velocity resolution and on instrumental noise (Padoan et al. 2001b). Here we have applied that improved SCF to a number of large ${ }^{13} \mathrm{CO}$ maps of molecular cloud complexes and obtained empirical correlations that can be used to test theoretical models. Of the theoretical models we have computed, some compare well with the empirical correlations and some do not, which shows that the SCF can be used as an effective tool to rule out inappropriate or unphysical models.

The empirical correlations we have obtained relate the values of $\alpha, S_{0}(1 \mathrm{pc})$, and $\sigma_{v}$ with each other. The $\alpha-\sigma_{v}$ correlation rules out the unphysical stochastic models (S2 and S4). Such models were found to produce spectral line profiles similar to observational ones by Dubinski, Narayan, \& Phillips (1995). They have also been used as models of the density field in molecular clouds by Stutzki et al. (1998) and to calibrate their principal component analysis by Brunt \& Heyer (2002). The SCF $\alpha-\sigma_{v}$ correlation shows that stochastic models are inappropriate to describe the structure of molecular cloud complexes.

Models not scaled with Larson-type relations (A1-A5, B1-B5) and models with larger-than-average column density (B1-B5, B1R-B5R) have also been compared with the empirical SCF correlations to show that incorrectly scaled models are readily ruled out by the SCF method.

The $S_{0}(1 \mathrm{pc})-\sigma_{v}$ and $\alpha-S_{0}(1 \mathrm{pc})$ correlations do not favor the model with equipartition of kinetic and magnetic energies (model E). Such models yield values of $S_{0}(1 \mathrm{pc})$ too small or values of $\alpha$ too large compared with the observational data, when seen in the direction parallel to the average 

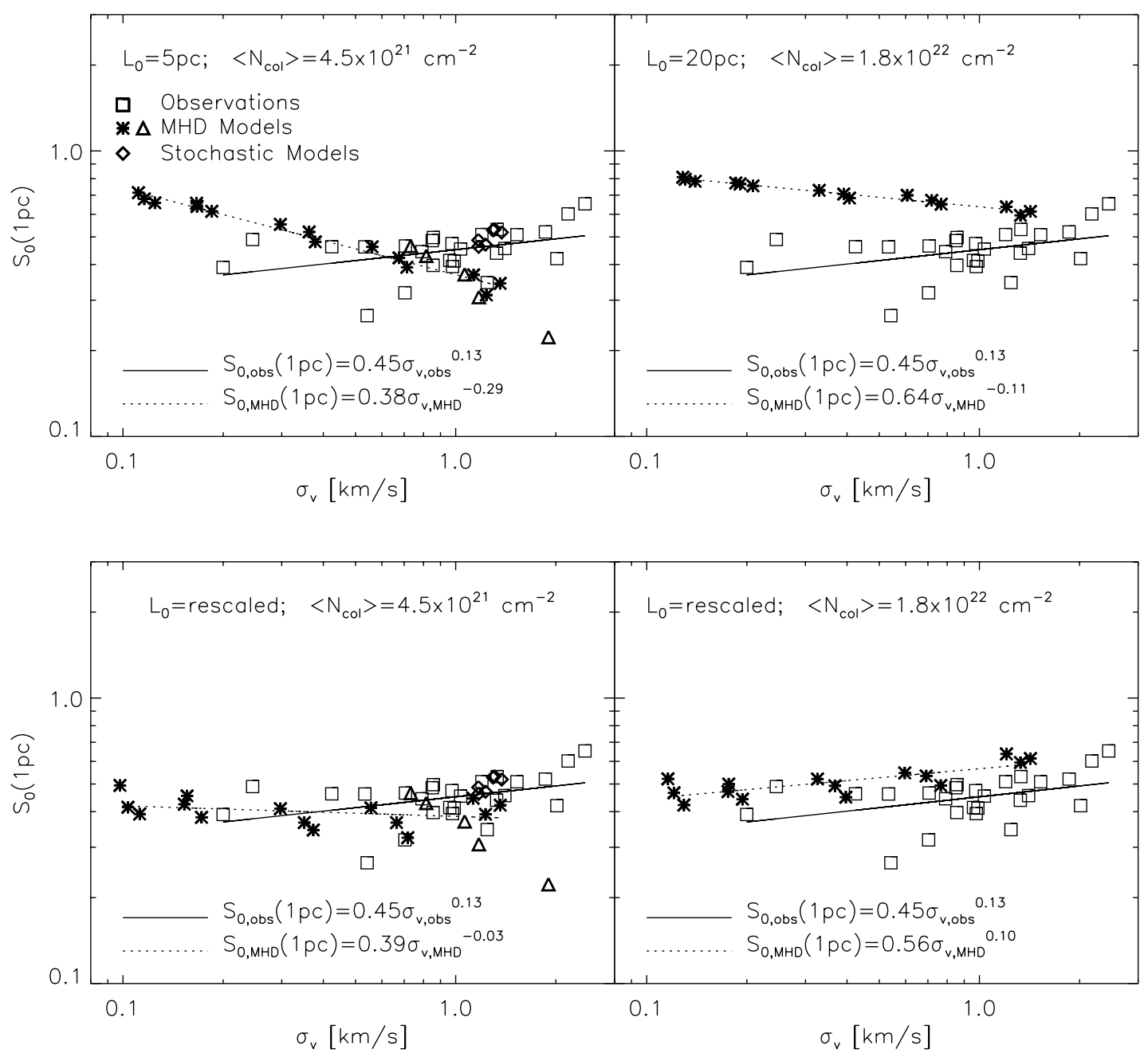

FIG. 6. - SCF value at 1 pe vs. velocity dispersion. Different panels show different models as in Fig. 5. Symbols are also as in Fig. 5.

magnetic field. Of the two diagonal directions, one is consistent with the data and the other is not.

A possible interpretation is that none of the observed regions have an average magnetic field oriented close to the direction of the line of sight. The equipartition model starts to be inconsistent with the observational data when seen along the diagonal directions, at an angle of 54.7 to the average magnetic field. The line of sight should be within such an angle to the magnetic field in approximately $40 \%$ of the cases, assuming random orientation of the average magnetic field in the observed regions.

An alternative interpretation is that all the observed regions have an average magnetic field strength smaller than in the equipartition model and consistent with superAlfvénic conditions. The super-Alfvénic models rescaled with Larson-type relations are in fact able to reproduce the empirical SCF correlations. However, the total number of truly independent directions on the sky in the present observational sample is still small. More regions should be studied to rule out the equipartition model based on the SCF results.

The analysis of the MHD models could, in principle, give different results if self-gravity were taken into account. However, the introduction of self-gravity is not expected to decrease the value of $\alpha$ and increase the value of $S_{0}(1 \mathrm{pc})$, as is necessary to make the equipartition model consistent with the observational $\alpha-S_{0}(1 \mathrm{pc})$ correlation. The main effect of self-gravity is the collapse of the densest regions, increasing the density contrast beyond the level due to the turbulence alone. This could slightly increase the value of $\alpha$, because we interpret the increase of $\alpha$ with $\sigma_{v}$ in the MHD models as being due to the increased compressibility of the turbulent flow. An increase in the value of $S_{0}(1 \mathrm{pc})$ is not expected because the local collapse of dense cores cannot increase the correlation between spectra at large distances [for a given value of $\alpha$, an increase in $S_{0}(1 \mathrm{pc})$ would correspond to an increase in the SCF at large spatial separation]. Nevertheless, the effect of self-gravity should be tested by including it in the numerical solution of the MHD equations. The numerical resolution should also be larger than in the present work to resolve the initial phase of the gravitational collapse of dense cores. We have only recently started to compute self-gravitating flows in a numerical mesh of $500^{3}$ cells, and their analysis will be presented in future works.

Padoan \& Nordlund (1999) have proposed that the dynamics of molecular clouds on large scales is consistent with super-Alfvénic turbulence and inconsistent with the equipartition model. In numerical simulations of superAlfvénic turbulence the average magnetic energy grows with time, even if flux is conserved (the average magnetic field is 

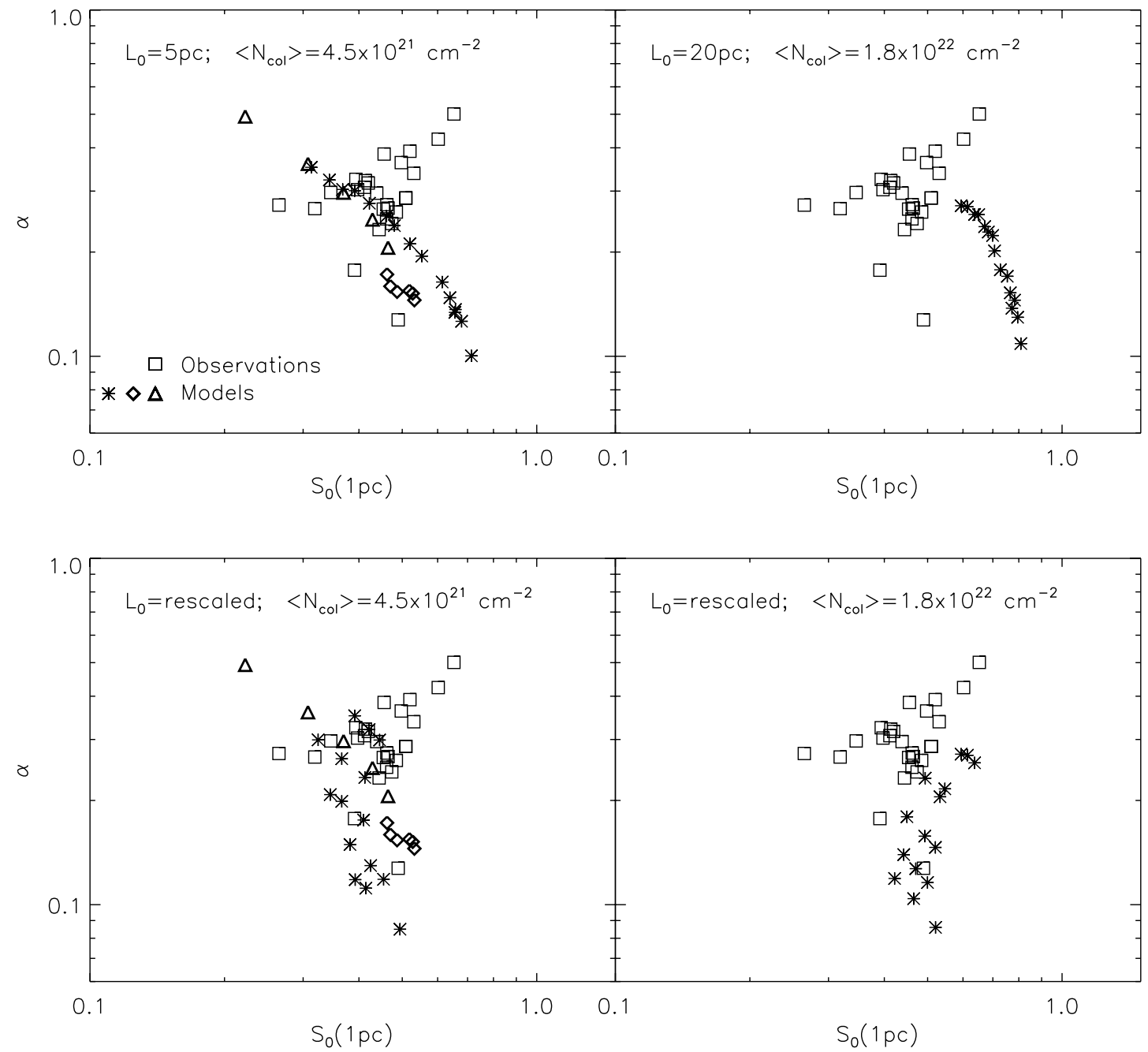

Fig. 7. - SCF slope vs. SCF value at 1 pc. Symbols and panels are as in Fig. 5.

constant). The magnetic field strength is increased locally mainly in regions of compression in supersonic turbulence and in part by stretching of field lines. Even if initial conditions are such that the turbulence is highly super-Alfvénic, magnetic pressure is often larger than thermal pressure in the postshock gas because of the amplification of the magnetic field components perpendicular to the shock direction. Equipartition of dynamic pressure, $\rho v^{2}$, and magnetic pressure, $B^{2} / 8 \pi$, is therefore achieved locally, but not necessarily over the whole flow. For example, in supersonic and superAlfvénic runs at a resolution of $250^{3}$, the ratio of volumeaveraged magnetic and dynamic pressures relaxes at a value $\left\langle P_{m}\right\rangle\left\langle\left\langle P_{d}\right\rangle \approx 0.12\right.$, starting from initial conditions with $\left\langle P_{m}\right\rangle_{\text {in }} /\left\langle P_{d}\right\rangle_{\text {in }} \approx 0.005$ (Padoan et al. 2003). Comparable values are found in the numerical experiments used in this work. The amplification of the magnetic field by the turbulence therefore does not alter the super-Alfvénic character of the flow.

The correlation between local magnetic field strength and gas density in supersonic and super-Alfvénic turbulence has a very large scatter and a well-defined upper envelope with $B \propto \rho^{0.4}$, both consistent with the observational data (Padoan \& Nordlund 1999; Ostriker et al. 2001; Passot \& Vázquez-Semadeni 2003). The largest values of the magnetic field strength are generally found in dense cores, but some dense cores may have a relatively weak magnetic field. However, dense cores assembled by turbulent shocks are not expected to have internal super-Alfvénic turbulence because of the dissipation of kinetic energy in the shocks and the amplification of the magnetic field in the compressed gas. Observational evidence for an approximate equipartition of turbulent and magnetic energy in dense cores would therefore not be inconsistent with the superAlfvénic character of the large-scale flow that assembles them.

The comparison between our theoretical models and the observational data could be improved if more regions with subsonic turbulence were available in the observational sample. Small velocity dispersion is found in small objects, according to Larson's velocity-size relation or to the power spectrum of turbulence. The spatial resolution in single-dish surveys is typically too low to sample a small object (fraction of a parsec) with a very large spectral maps (several thousands of spectra). The only exceptions in the observational sample used in this work are L1512 and L134a. These two large maps of nearby clouds with very low velocity dispersion were obtained by Falgarone et al. (1998) as part of their IRAM key project, focused on regions of relatively low column density at the edges of molecular cloud complexes. 
Maps of large regions with very large velocity dispersion are instead more easily obtained from observations than in numerical simulations. Assuming a gas kinetic temperature of the order of $10 \mathrm{~K}$, a line-of-sight (one-dimensional) velocity dispersion in excess of $2 \mathrm{~km} \mathrm{~s}^{-1}$ corresponds to a sonic rms Mach number of the flow $M_{S} \geq 20$. In the present work we have not computed numerical flows with $M_{S}>10$, since that would require a larger numerical resolution (the density contrast grows linearly with the Alfvénic Mach number and therefore with the value of $M_{S}$ if the average magnetic field strength is not varied). For this reason, the models do not reach the largest values of $\sigma_{v}, \alpha$, and $S_{0}(1 \mathrm{pc})$ obtained from the observations (from the maps of the Rosette molecular cloud complex). The progression of models toward increasing values of $M_{S}$ suggests that a model with $M_{S} \geq 20$ would likely fit the observed $\alpha-S_{0}(1 \mathrm{pc})$ values found in the Rosette molecular cloud complex, where the observed velocity dispersion is in excess of $2 \mathrm{~km} \mathrm{~s}^{-1}$. This is illustrated in Figure 8. The top panel of Figure 8 shows the $\alpha-S_{0}(1 \mathrm{pc})$ for the observational data. The shaded area shows the range of values covered by the theoretical models $\mathrm{A} 1 \mathrm{R}-\mathrm{A} 5 \mathrm{R}$. The bottom panel shows the same plot for the models A1RA5R. Each diagonal segment connects the values for the

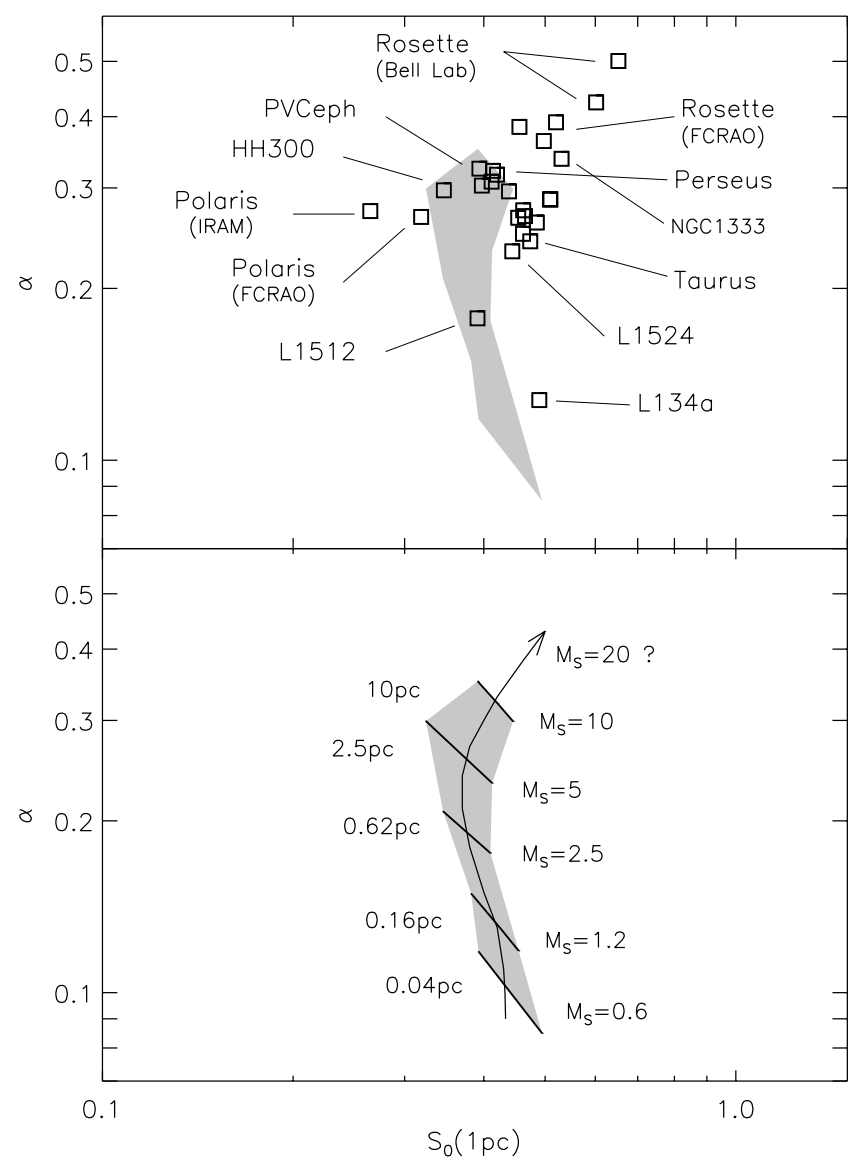

FIG. 8.-Top: Values of $\alpha$ and $S_{0}(1 \mathrm{pc})$ from the observations. Some of the symbols are labeled with the region name. The shaded area shows the range of values covered by the models A1R-A5R. Bottom: Same shaded area as in the top panel. Diagonal segments show the range of values of $\alpha$ and $S_{0}(1 \mathrm{pc})$ for the three directions of each model. The rms sonic Mach number of the corresponding model is given on the right-hand side of each segment, while the value of the linear size is given on the left-hand side. The arrow indicates the progression of models toward larger values of sonic Mach number, $M_{S}$. three directions of each model. The values of $M_{S}$ and $L_{0}$ of the models are also given in the plot. The arrow marks the direction of increasing $M_{S}$, suggesting that models with $M_{S} \approx 20$ may fit the observations with the largest velocity dispersion.

\section{SUMMARY AND CONCLUSIONS}

In the present work we have computed the SCF of spectral maps of molecular cloud complexes and regions within them, observed in the $J=1-0$ transition of ${ }^{13} \mathrm{CO}$. We have found that the SCF is a power law over approximately an order of magnitude in spatial separation. The power-law slope of the SCF, $\alpha$, its normalization, $S_{0}(1 \mathrm{pc})$, and the spectral line width averaged over the whole map, $\sigma_{v}$, have been computed for all the observational maps. We have obtained empirical correlations between these quantities and have proposed to use them to test the validity of theoretical models of molecular clouds.

Theoretical models of spectral line maps have been generated by computing the radiative transfer through the numerical solutions (density and velocity fields) of the MHD equations, for turbulent flows with different values of the rms sonic and Alfvénic Mach numbers, and also through stochastic density and velocity fields with different power spectra. Super-Alfvénic MHD models rescaled according to Larson-type relations are in the best agreement with the empirical correlations. Unphysical stochastic models are instead ruled out. MHD models with equipartition of magnetic and kinetic energy of turbulence do not reproduce the observational data when their average magnetic field is oriented approximately parallel to the line of sight. Finally, MHD models not rescaled according to Larson-type relations are also inconsistent with the observational data.

We cannot exclude the possibility that different physical models for the dynamics of molecular clouds, or even unphysical models, that we have not tested here would satisfy the empirical correlations found in this work. However, we have shown that the SCF method is able to rule out certain unphysical or incorrectly scaled models. Reproducing these SCF results should be considered as a necessary (but not sufficient) condition for the validity of theoretical models describing the structure and the dynamics of molecular clouds. Models for which the SCF or similar statistical tests cannot be computed to allow a quantitative comparison with observed spectral maps cannot be legitimately evaluated.

The comparison between theory and observations presented in this work requires significant computational resources. Numerical simulations of three-dimensional turbulent flows must be run at large resolution and the radiative transfer has to be computed in three dimensions in order to generate synthetic spectral maps of the observed molecular transitions. The type of models and the physical parameters investigated in this work are therefore limited to a few significant cases. Future work should investigate the SCF of a larger variety of models, including different magnetic field intensities and flows with gravitationally collapsing cores.

We are grateful to Eve Ostriker and Jim Stone for helpful comments on our model-data comparison. The referee report by Enrique Vázquez-Semadeni has also contributed to improving this work. This work was supported by an 
NSF Galactic Astronomy grant to A. A. G. The work of P. P. was partially performed while P. P. held a National Research Council Associateship award at the Jet Propulsion
Laboratory, California Institute of Technology. M. J. acknowledges the support of the Academy of Finland grant 1011055.
Adler, D. S. \& Roberts, W. W 1992, ApJ, 384,95

Arce, H. G., \& Goodman, A. A. 2001, ApJ, 554, 132

Ballesteros-Paredes, J., \& Mac Low, M. 2002, ApJ, 570, 734

Ballesteros-Paredes, J., Vázquez-Semadeni, E., \& Goodman, A. A. 2002, ApJ, 571, 334

Ballesteros-Paredes, J., Vázquez-Semadeni, E., \& Scalo, J. 1999, ApJ, 515, 286

Bensch, F., Stutzki, J., \& Ossenkopf, V. 2001, A\&A, 366, 636

Bernes, C. 1979, A\&A, 73, 67

Blitz, L., \& Stark, A. A. 1986, ApJ, 300, L89

Blitz, L., \& Williams, J. P. 1997, ApJ, 488, L145

Brunt, C. M., \& Heyer, M. H. 2002, ApJ, 566, 276

Dubinski, J., Narayan, R., \& Phillips, T. G. 1995, ApJ, 448, 226

Falgarone, E., Lis, D. C., Phillips, T. G., Pouquet, A., Porter, D. H., \& Woodward, P. R. 1994, ApJ, 436, 728

Falgarone, E., Panis, J.-F., Heithausen, A., Pérault, M., Stutzki, J., Puget, J.-L., \& Bensch, F. 1998, A\&A, 331, 669

Falgarone, E., Puget, J. L., \& Pérault, M. 1992, A\&A, 257, 715

Flower, D. R., \& Launay, J. M. 1985, MNRAS, 214, 271

Gill, A. G., \& Henriksen, R. N. 1990, ApJ, 365, L27

Green, S., \& Thaddeus, P. 1976, ApJ, 205, 766

Heyer, M. H., \& Schloerb, F. P. 1997, ApJ, 475, 173

Hobson, M. P. 1992, MNRAS, 256, 457

Houlahan, P., \& Scalo, J. 1992, ApJ, 393, 172

Issa, M., MacLaren, I., \& Wolfendale, A. W. 1990, ApJ, 352, 132

Juvela, M. 1997, A\&A, 322, 943

Kegel, W. H. 1989, A\&A, 225, 517

Kim, S., Dopita, M. A., Staveley-Smith, L., \& Bessell, M. S. 1999, AJ, 118, 2797

Kim, S., Staveley-Smith, L., Dopita, M. A., Freeman, K. C., Sault, R. J.,

Kesteven, M. J., \& McConnell, D. 1998, ApJ, 503, 674

Kleiner, S. C., \& Dickman, R. L. 1985, ApJ, 295, 466

. 1987, ApJ, 312, 837

Langer, W. D., Wilson, R. W., \& Anderson, C. H. 1993, ApJ, 408, L45

Larson, R. B. 1981, MNRAS, 194, 809

Lazarian, A., Pogosyan, D., Vázquez-Semadeni, E., \& Pichardo, B. 2001, ApJ, 555, 130

\section{REFERENCES}

Lis, D. C., Pety, J., Phillips, T. G., \& Falgarone, E. 1996, ApJ, 463, 623

Loren, R. B. 1989, ApJ, 338, 925

Mac Low, M.-M., \& Ossenkopf, V. 2000, A\&A, 353, 339

Miesch, M. S., \& Bally, J. 1994, ApJ, 429, 645

Miesch, M. S., \& Scalo, J. M. 1995, ApJ, 450, L27

Miesch, M. S., Scalo, J., \& Bally, J. 1999, ApJ, 524, 895

Mizuno, A., Onishi, T., Yonekura, Y., Nagahama, T., Ogawa, H., \& Fukui, Y. 1995, ApJ, 445, L161

Myers, P. C., \& Goodman, A. A. 1988, ApJ, 329, 392

Ossenkopf, V. 2002, A\&A, 391, 295

Ostriker, E. C., Stone, J. M., \& Gammie, C. F. 2001, ApJ, 546, 980

Padoan, P., Bally, J., Billawala, Y., Juvela, M., \& Nordlund, A. 1999, ApJ, 525,318

Padoan, P., Jimenez, R., Nordlund, Å., Boldyrev, S. 2003, Phys. Rev. Lett., submitted

Padoan, P., Juvela, M., Bally, J., \& Nordlund, Å. 1998, ApJ, 504, 300

.2000, ApJ, 529, 259

Padoan, P., Juvela, M., Goodman, A. A., \& Nordlund, Å. 2001a, ApJ, 553, 227

Padoan, P. \& Nordlund, ̊̊. 1999, ApJ, 526, 279

Padoan, P., Rosolowsky, E. W., \& Goodman, A. A. 2001b, ApJ, 547, 862

Passot, T., \& Vázquez-Semadeni, E. 2003, A\&A, 398, 845

Pichardo, B., Vázquez-Semadeni, E., Gazol, A., Passot, T., \& BallesterosParedes, J. 2000, ApJ, 532, 353

Rosolowsky, E. W., Goodman, A. A., Wilner, D. J., \& Williams, J. P. 1999, ApJ, 524, 887

Scalo, J. 1990, in Physical Processes in Fragmentation and Star Formation, ed. R. Capuzzo-Dolcetta, C. Chiosi, \& A. Di Fazio (Dordrecht: Kluwer), 151

Scalo, J. M. 1984, ApJ, 277, 556

Stutzki, J., Bensch, F., Heithausen, A., Ossenkopf, V., \& Zielinsky, M. 1998, A\&A, 336, 697

Stutzki, J., \& Güsten, R. 1990, ApJ, 356, 513

Vázquez-Semadeni, E., Ballesteros-Paredes, J., \& Rodríguez, L. F. 1997, ApJ, 474, 292

Williams, J. P., De Geus, E. J., \& Blitz, L. 1994, ApJ, 428, 693 